\title{
Medico-socio-economic variables of trauma presented to the pediatric emergencies: A monocentric study
}

Myriam Amm $^{1}$, Yara salameh ${ }^{1}$, Zaki Ghorayeb $^{1}$, Hala Feghali ${ }^{1}$, Georges Nicolas ${ }^{1}$, Mode AlOjaimi ${ }^{2}$ and Marie Claude Fadous Khalife ${ }^{*}$

${ }^{1}$ Holy Spirit University of Kaslik, UH-Notre Dame Des Secours, Lebanon

${ }^{2}$ Balamand University, Lebanon

\begin{abstract}
Introduction: Most of the pediatric cases seen in the emergency department are due to traumatic causes, and this remains a major cause of death. Therefore, a retrospective study of 446 traumatized children in pediatric emergencies (September 2016-February 2017) at Notre-Dame de Secours hospital was conducted over 6 months in order to create a clearer vision about this matter.

Results: Boys were the more affected by trauma with a percentage of $66.3 \%$, an average age of 7.8 years and a frequency of $67 \%$ for falling with a predominance for the upper limbs injury. School trauma accounts for $24.3 \% .56 .7 \%$ of the imaging studies were positive. The average billing was 320,000 L.L. The average waiting time was 1.1 hours. Only $6.2 \%$ of the trauma patients were admitted and $1.82 \%$ transferred to the operating room. The majority of admissions were head trauma.

Conclusion: Child injuries have so far been a medical and socio-economic burden since they present high mortality and morbidity rates. This is a pilot study that should be extended throughout Lebanon to have objective feedback data about pediatric trauma. This will enable us to improve systems of care for these patients and to establish adequate prevention systems.
\end{abstract}

\section{Introduction}

Being curious about their environment, children engage in dangerous activities that may cause severe trauma. Around the world, every hour, 100 children are victims of preventable accidental injuries, and every year, one million children under 18 years old die accidentally [1]. Trauma may lead to bad consequences: mortality, disability and post-traumatic school regression, which can be prevented by developing recommendations, based on epidemiological studies [1-4].

Over the last 20 years, Western societies have focused on this topic by conducting studies and amending legislation to establish individual and public safety standards [1-3]. However, research and publications remain rare in the Middle East and specifically in Lebanon. In many Lebanese hospitals, the pediatric emergencies are mixed with adult emergencies and so is the data [2]. Unfortunately, most Lebanese hospitals do not have specialized pediatric emergency team and services.

\section{Materials and Methods}

The retrospective study was conducted between September 2016 and February 2017. The cases were collected from the pediatric emergency archives of Notre Dame de Secours (Jbeil-Byblos). 446 patients under 18 years old were recruited in this study. The studied variables were: age, sex, nationality, place of residence, time spent in the emergency room, location, type of trauma, area affected, para clinical examinations performed and their results, billing and final patient disposition.

\section{Statistical analysis}

The data was analyzed using SPSS (Statistical Package for Social Science) software version 20. The statistics were calculated using percentages for qualitative variables and on average \pm standard deviation for quantitative variables in order to analyze the basic characteristics, clinical and Paraclinical parameters of study participants. The "Chisquare", "Student t" and "one- way Anova" Tests and other nonparametric tests were used when one of the two groups had a $\mathrm{N}<30$ number (Fisher exact Test, Kruskall Wallis Test).

\section{Results}

The percentage of boys with trauma was $66.3 \%$ with an average age 7.8 years. $75 \%$ of the accidents occurred at home. Lebanese nationality was predominant with $98.6 \%$. Most common cause of trauma was falling down. The most affected body regions were the upper limbs, the face, the lower limbs and the head. In $56.7 \%$ of cases, medical imaging was requested where only $28.4 \%$ of them were positive. The average time spent at the emergencies was 1.1 hours with a maximum of 9 hours waiting. $91.9 \%$ of the traumatized patients were discharged home while $6.2 \%$ were admitted and $1.8 \%$ were transferred to the operating room. Finally, the average of the bill price was LBP 319,874.35 ( USD 233.00). A positive correlation was found between trauma of superior limbs, age $(\mathrm{p}=0.001)$ and the sex of the child $(\mathrm{p}=0.04)$. (Tables 1 and 2, Figure 1)

\section{Discussion}

The majority of affected children were boys, which is consistent with Musharrafieh et al. Wang et al. (74.6\%), and Nuwayhid et al. (68\%) $[2,3,5,6]$ This difference could be due to the aggressive games of boys compared to the relatively safer games of the girls $[1,2]$.

${ }^{\star}$ Correspondence to: Marie Claude Fadous Khalife, Holy Spirit University of Kaslik, UH-Notre Dame Des Secours, Lebanon, E-mail: mclaude454@hotmail.com

Key words: trauma, fall, drowning, road accident, burn, intoxication

Received: August 10, 2018; Accepted: August 22, 2018; Published: August 27, 2018 
Table 1. Characteristics of the participants

\begin{tabular}{|c|c|c|c|}
\hline \multicolumn{2}{|l|}{ Independent variables } & \multirow{2}{*}{$\begin{array}{l}\text { Number } \\
289\end{array}$} & \multirow{2}{*}{\begin{tabular}{|l} 
Percentage \\
66.3
\end{tabular}} \\
\hline$S_{a}$ & Boy & & \\
\hline sex & Girl & 147 & 33.7 \\
\hline \multirow{3}{*}{ Nationality } & Lebanese & 430 & 98.6 \\
\hline & Syrian & 5 & 1.1 \\
\hline & Iraqi & 1 & 0.2 \\
\hline \multirow{5}{*}{ Mechanisms } & Fall & 180 & 67.2 \\
\hline & Car accident & 9 & 3.4 \\
\hline & Burn & 4 & 1.5 \\
\hline & Poisoning/Foreign Body & 22 & 8.2 \\
\hline & Other & 53 & 19.8 \\
\hline \multirow{3}{*}{ Paraclinical exam } & Radio & 229 & 63.3 \\
\hline & Scan & 18 & 5 \\
\hline & None & 12 & 3.3 \\
\hline \multirow{2}{*}{ Paraclinical exam } & Yes & 247 & 56.7 \\
\hline & No & 189 & 43.3 \\
\hline \multirow{2}{*}{ Paraclinical exam result } & Positive & 67 & 28.4 \\
\hline & Negative & 169 & 71.6 \\
\hline \multirow{3}{*}{ Hospitalization or discharge } & Discharge & 398 & 91.9 \\
\hline & Admissions & 27 & 6.2 \\
\hline & Emergent operation & 8 & 1.8 \\
\hline \multirow{2}{*}{ Place of the accident } & Home & 240 & 75.7 \\
\hline & School & 77 & 24.3 \\
\hline \multirow{7}{*}{ Localization of the injury } & Head & 79 (41 head trauma) & 18.9 \\
\hline & Face & 102 & 24.3 \\
\hline & Thorax & 4 & 1 \\
\hline & Abdomen and pelvis & 4 & 1 \\
\hline & Back & 8 & 1.9 \\
\hline & Superior member & 132 & 31.5 \\
\hline & Inferior member & 90 & 21.5 \\
\hline Age & \multicolumn{3}{|l|}{$7.8 \pm 4.65$} \\
\hline Waiting time & \multicolumn{3}{|c|}{$1.1 \pm 0.87$, Min $=0.16$ hours $/ \mathrm{Max}=9 \mathrm{~h}$} \\
\hline Residency & \multicolumn{3}{|c|}{ Jbeil-Byblos $=16.3 \%$ Amchit $=14.2 \%$ Halat $=6.2 \%$ Batroun $=4.8 \%$ Mastita $=4.6 \%$} \\
\hline Billing & \multicolumn{3}{|c|}{$319,874.35 \mathrm{LBP} \pm 709,822.543 \mathrm{LBP}, \mathrm{Min}=50,000 \mathrm{LBP} / \mathrm{Max}=9,444,518 \mathrm{LBP}$} \\
\hline
\end{tabular}

The qualitative variables are expressed in percentage and the quantitative variables in average \pm Standard deviation, Min: Minimum, Max: Maximum, LBP: Lebanese Pounds

Table 2. Association between the localisation of the injury and the independent variables

\begin{tabular}{|c|c|c|c|c|}
\hline \multicolumn{2}{|c|}{ Independent variables } & \multicolumn{2}{|l|}{ Groups } & P-value \\
\hline \multicolumn{5}{|c|}{ Superior limb } \\
\hline & & Yes & No & \\
\hline \multicolumn{2}{|l|}{ Age } & $8.94 \pm 4.55$ & $7.3 \pm 4.61$ & $0.001 *$ \\
\hline \multirow{2}{*}{ Sex } & Boy & 27 & 73 & \multirow{2}{*}{$0.04 *$} \\
\hline & Girl & 36.7 & 63.3 & \\
\hline
\end{tabular}

\section{Injuries billing amounts}

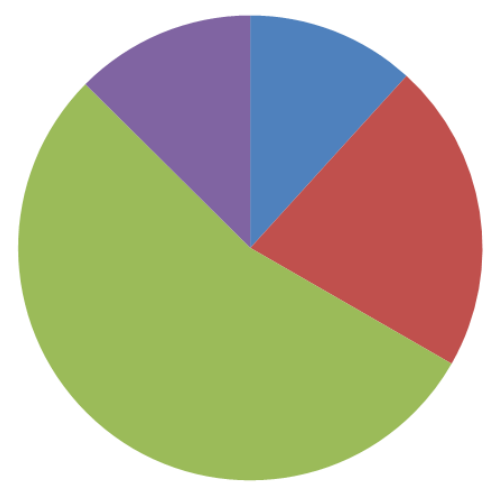

w poisoning/foreign body ingestion ( LBP 257864.5)

a burns( LBP 472875.3)

m car accident ( LBP 1187825)

a fall( LBP275989.4)

Figure 1. The highest billing costs go for road and burns accidents

Falling down was the most common trauma type: these results are also compatible with those of Musharrafieh et al. The WHO World Report on Injury Prevention (2008), however, does not correlate with those of Nuwayhid et al. for whom falls were ranked second after perforations and sharps wounds $(42 \%)[1,2,6]$. The number of injuries caused by firearms $(0.66 \%)$ or other knives $(3.34 \%)$ is very rare in Jbeil, while Musharrafieh and Nuwayhid have rates of $12.3 \%$ and $42 \%$ for firearm injury [2,6]. For Gerbaka et al. in 1987/88, firearms accounted for $21.3 \%$ of reported accidents $[2,6,7]$. The results of Gerbaka et al. correspond to a period when Lebanon was at war.

Road traffic accidents (3.4\%) in third place are lower than in Musharrafieh et al. (5.2\%), compared to $8.9 \%$ at Gerbaka et al. This decrease could be due to better parental supervision and media campaigns for prevention as well as the increased number of NGOs campaigning for road safety in Lebanon [2,7].

Burns accounted for only $1.5 \%$ of cases, similar to Musharrafieh et al. (1.3\%) [2]. While this incidence remains high, reaching 29 to $51 \%$ in some countries [1]. Incidence of burn was similar in both sexes, which is not compatible with Zahid et al. and the WHO World Report on Injury Prevention. The latter explains that the predominance of women was due to girls' interest in cooking [8].

Only two cases of ski trauma are noted, this is explained by the presence of a hospital near the ski resorts taking care of these patients. 
On the other hand, no sexual assault or abuse has been recorded and this could be explained either by the lack of experience of health professionals or by Lebanese taboo issues. This is the reason why a child protection unit was created at the Notre-Dame de Secours hospital.

The most affected parts are the upper limbs $(31,5 \%)$, result compatible with those of Nuwayhid et al. (39\%) and Musharrafieh et al. $(38 \%)[2,6]$.

The average age of involvement of members is 9-10 years old, age of preadolescence.

Fractures of the limbs may be complicated by post-traumatic osteoarthritis [9]. It is therefore imperative to protect the upper and lower limbs at this age during sports activities.

Injuries occurring in school represent $24.3 \%$. The average age of affected children is 10 years with a predominance for boys and for falling. For this reason, school institutions need to be more vigilant about monitoring boys. Similarly, parents and other institutions taking care of children should meticulously supervise children [1].

These latter results are similar to those of Sundblad et al. except for sex for which girls are also victims during sports activities [8].

The 2 major causes of hospitalization are fractures and head trauma due to falls and road accidents. The majority of head injuries are benign [9]. For Nuwayhid et al. the 3 major causes of admission are public road accidents, poisoning, and falls [6].

The average patients' age in our study was 7.8 years, which is not compatible with that of Nuwayhid et al. where the average is between 1 and 4 years [6]. However, it is compatible with that of Wing et al. for whom the average age is 10.8 years.

In order of frequency, road accidents came first, followed by falling, poisonings and burns. This is related to the psychomotor development of the child because as he grows up, he becomes more autonomous and can easily escape the supervision of his parents [10].

The waiting time in the emergency department was 1.1 hours, similar to Musharrafieh et al. (62 min) [2]. It is a relatively acceptable and short waiting time showing adequate and prompt care.

In Lebanon the financial aspect of medical care is an important burden. The average cost of care for radiologic studies in the emergency is 406,074 L.L (\$ 266) compared to $204,105 \mathrm{~L}$. L (\$ 136) when no radiologic studies were ordered.

In $56.7 \%$ of cases, medical imaging was requested: $71.6 \%$ were negative and their average cost was $246.139 .90 \mathrm{~L}$ (\$164).

The financial burden of these radiological studies on the private patient and on third-party payers should not be ignored. Accordingly, it is necessary to avoid the abusive demands of radiological examinations, a more rigorous clinical examination must be adapted. In addition, it would be interesting to conduct a study to see if there is a positive correlation between the abuse of these requests for radiological examinations and the presence of a supplementary insurance contract.

Road accidents generated the highest average cost followed by burns, falling and poisonings. This is due to the delicate, multidisciplinary and medico-legal management of poly trauma patients who are by definition patients with at least three severe lesions.

Lower limb trauma, followed by head trauma, is the most expensive traumas. Indeed, half of the fractures of the lower limbs required casting with a hospitalization frequency of $3 \%$. On the other hand, $32 \%$ of head injuries were severe enough to require a brain scan with $55.56 \%$ hospitalization rate.

It is known that $30 \%$ of Lebanese are covered by the CNSS (National Social Security Fund), $45.5 \%$ by the Ministry of Health, $8 \%$ by private insurance and the remaining $16.5 \%$ are divided between the Lebanese army and other government institutions [11]. The majority of patients $(66 \%)$ attending our emergencies are covered by private insurance and $34 \%$ are either supported by the CNSS and the Ministry of Health, or private. To explain these results, we can assume that most traumatized patients receiving financial support by the public services have gone to regional peripheral hospitals and dispensaries even if these structures do not have pediatric emergencies. For these reasons, the government should change its reimbursement policy to allow all Lebanese to benefit from the best care especially in case of children traumatism.

\section{Limitations}

The study was limited to a winter period without covering the spring and summer months. As the winter season this year had few snowing episodes, few ski trauma cases were seen.

No cases of sexual assault or abuse have been recorded.

\section{Conclusion}

Child trauma remains a medical and socio-economic burden. It is associated with high mortality and morbidity rates. This pilot study should be extended to all Lebanon in order to provide objective feedback regarding the pediatric patient care standards and to establish adequate rules of prevention and protocols for improvement.

\section{References}

1. World Health Organisation (2008) World Report on Child Injury Prevention. [Crossref]

2. Musharrafieh U, Rahi AC, Taha A (2011) Profile of injured patients presenting to a tertiary hospital in a developing country. J Med Liban 59: 191-196.

3. Les traumatismes chez les enfants et les jeunes québécois âgés de 18 ans et moins: éta de situation.

4. Pape HC, Lefering R, Butcher N, Peitzman A, Leenen L, et al. (2014) The definition of polytrauma revisited: An international consensus process and proposal of the new "Berlin definition." J Trauma Acute Care Surg 77: 780-786. [Crossref]

5. Wang H, Yu H, Zhou Y, Li C, Liu J, et al. (2017) Traumatic fractures as a result of falls in children and adolescents: A retrospective observational study. Medecine(Baltimore) 96: e7879. [Crossref]

6. Iman A. Nuwayhid, Mona Al-Kouatly Kambris, Myrna Mahfoud (2002) Childhood injuries in the city of Beirut: the experience of three major emergency services. Lebanese Science Journal 3: 29-41.

7. Gerbaka B, Rassi P, Chaib-Ghosn A, Beaufils F, Akatcherian C (1996) Accidents in children. Retrospective epidemiological study of 1671 observations collected at the Hôtel-Dieu Beirut. J Med Liban 44: 209-214.

8. Sundblad G, Saartok T, Engstrom LM, Renström P (2005) Injuries during physica activity in school children. Scand J Med Sci Sports 15: 313-323. [Crossref]

9. Fractures of the lower extremity of the radius. Faculty of Medicine Pierre and MarieCurieAccédé en janvier 2018

10. Cemea-Infop E. Javouhey (2007) Psychology and child development. Child victims of unsafe roads: epidemiology of trauma and sequelae. Other [q-bio.OT]. Claude Bernard University - Lyon I.

11. Cemea-Infop E. Javouhey (2007) Psychology and child development. Child victims of unsafe roads: epidemiology of trauma and sequelae. Other [q-bio.OT]. Claude Bernard University - Lyon I.

Copyright: (C2018 Amm M. This is an open-access article distributed under the terms of the Creative Commons Attribution License, which permits unrestricted use, distribution, and reproduction in any medium, provided the original author and source are credited. 